\title{
Probing electron conduction at the microscopic level in percolating nanocomposites by conducting atomic-force microscopy
}

\author{
E. Z. Luo* and I. H. Wilson \\ Materials Technology Research Center, The Chinese University of Hong Kong, Shatin, N.T., Hong Kong, China \\ X. Yan \\ Department of Physics, Hong Kong University of Science and Technology, Clear Water Bay, Kowloon, Hong Kong, China \\ J. B. Xu \\ Materials Technology Research Center, The Chinese University of Hong Kong, Shatin, N.T., Hong Kong, China \\ (Received 28 October 1997; revised manuscript received 13 February 1998)

\begin{abstract}
We have studied systematically the conductance distribution in nanocomposite $\mathrm{Ni}_{x}\left(\mathrm{SiO}_{2}\right)_{1-x}$ thin films with $x$ near the percolation threshold $x_{c}$ by conducting atomic-force microscopy. We have experimentally demonstrated that there exists power-law and exponential dependences of the conductance distribution in low- and high-conductance regions, respectively. The conductance distribution was found to be independent of the bias, indicating that Ohmic behavior was satisfied at the microscopic level. Quantitative analysis further suggests that the tunneling effect leads to the power-law distribution of conductance in the low-conductance region. [S0163-1829(98)50624-8]
\end{abstract}

There is renewed interest in magnetic metal-insulator nanocomposite materials, due to the discovery of giant magnetoresistance ${ }^{1,2}$ and giant Hall effect. ${ }^{3}$ All these enhanced physical properties are related to electron conduction in the vicinity of the percolation threshold. Macroscopic measurements have made a considerable contribution to our understanding of the percolation phenomenon. The classical percolation picture was proven to be sufficient to describe many percolating systems. ${ }^{4,5}$ However, when the constituent metal particles are of nanometer size, phenomena such as the giant Hall effect and small and temperature insensitive electric power in percolating $\mathrm{Ni}-\mathrm{SiO}_{2}{ }^{3,6,7}$ and $\mathrm{Fe}-\mathrm{SiO}_{2},{ }^{7}$ cannot be explained by any version of the classical models. In addition, universality is also not applicable to such systems. Theoretically, nonuniversality has to be explained by a continuous percolation model, which explicitly relates the critical exponents to the details of conductance distribution in a system. ${ }^{8,9,10}$ Microscopically, a percolating metal-insulator composite consists of a number of conducting paths (CP's), each of them can be characterized by a conductance $g$. Obviously, a statistical probability distribution $P(g)$ can describe such disordered systems. Feng et al. ${ }^{8}$ and Balberg ${ }^{9,10}$ predicated that $P(g) \propto g^{-\gamma}$ as $g \rightarrow 0$. However, the conductance distribution has never been experimentally measured. Clearly, it is highly desirable to study electron conduction down to individual CP's and probe the conductance distribution at the microscopic level in order to understand conduction mechanisms and the macroscopic properties. Attempts along this line include electric-force microscopy (EFM) studies of conducting networks in a black carbon-insulator system, ${ }^{11}$ and the conducting atomic-force microscopy (CAFM) studies on $\mathrm{Ni}_{-} \mathrm{SiO}_{2}$ and $\mathrm{Fe}-\mathrm{SiO}_{2} \cdot{ }^{12-13}$ Moreover, understanding the conduction mechanism will help us to understand other related phenomena in these systems. ${ }^{14-16}$ In this paper, we present a systematic study of the conductance distribution in $\mathrm{Ni}_{x}\left(\mathrm{SiO}_{2}\right)_{1-x}$ nanocomposite films with $x$ around $x_{c}$ by C-AFM. We experimentally demonstrate the existence of the power law and the exponential distribution of conductance in low- and high-conductance region, respectively.

1- $\mu$ m-thick $\mathrm{Ni}_{x}\left(\mathrm{SiO}_{2}\right)_{1-x}$ films were prepared by RF cosputtering of $\mathrm{Ni}$ and $\mathrm{SiO}_{2}$ on silicon wafers at $450 \mathrm{~K}$, details of the sample preparation have been described elsewhere. ${ }^{3}$ The C-AFM used in our experiments was constructed by modifying the commercial NanoscopeIII AFM (Digital Instruments, Inc.) with a homemade pre-amplifier and a bias unit with maximum current range of $\pm 10 \mathrm{nA}$. The noise of the pre-amplifier and the bias unit is below $\pm 1.5 \mathrm{pA}$ and 0.1 $\mathrm{mV}$ respectively. The $\mathrm{C}$-AFM works at contact, constant force, and constant bias mode. Commercial $\mathrm{Si}_{3} \mathrm{~N}_{4}$ cantilevers coated with a $10 \mathrm{~nm}$ Cr layer plus $100 \mathrm{~nm}$ of gold were used. The loading force was calibrated at about $70 \mathrm{nN}$. The experiments were carried out in a dry nitrogen environment while the relative humidity was maintained at $17.5 \%$ during measurements. When the AFM tip rasters a surface of metalinsulator composite, the tip contacts either the metallic or the insulating phase. When the tip contacts a metal particle, which is part of the conducting networks $(\mathrm{CN})\left(x>x_{c}\right)$ that extends to the surface, then electrons can flow between the sample and the tip when a bias is applied. For $x<x_{c}$, but near the percolating threshold, tunneling leads to a detectable current. ${ }^{12}$ Figure 1 shows typical electric current image for $x=0.532$ at a bias of $9.2 \mathrm{mV}$. Here $x$ is the volume fraction of the metallic phase. All the experimental results presented here were obtained with the same tip. The current spikes are part of CP's formed in the bulk that extend to the surface. ${ }^{12,13}$ The current images are typical and reproducible. All the data discussed here and the conductance distributions were obtained in a $1 \mu \mathrm{m} \times 1 \mu \mathrm{m}$ area. Such a scan area was chosen based on the compromise of spatial resolution and good statistics. Quantitatively, by averaging the current images at different biases, one can obtain the averaged $I-V$ 


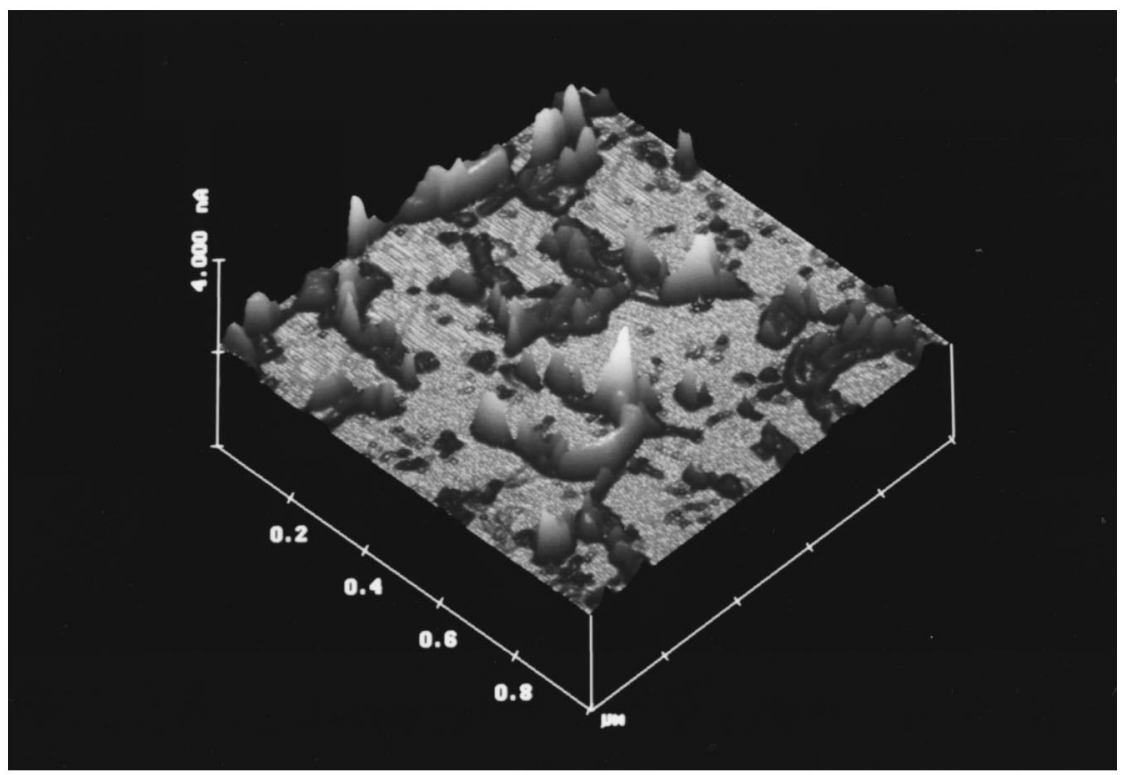

FIG. 1. A typical electric current image obtained on a sample with $x=0.532$ at a bias of $14.5 \mathrm{mV}$.

plots for different samples, as illustrated by Fig. 2. The slope of the averaged $I-V$ plots is the average conductance $G_{\mathrm{av}}$. From Fig. 2 one can clearly see that the averaged $I-V$ is linear for all samples, indicating an Ohmic conduction at low bias. The inset of Fig. 2 shows the dependence of the $G_{\text {av }}$ on $x$, which shows a rapid increase of $G_{\mathrm{av}}$ for $x>0.532$. It indicates that the percolation threshold $x_{c}$ is around 0.532, which agrees quite well with macroscopic measurements. ${ }^{3}$

In addition to measuring the average conductance by image analysis, one can also obtain the current distribution $P(I)$, the probability of current $I$. At each point in the current image, we assume that $I=g V$ holds. If variable $I$ is divided by the bias voltage $V$, i.e., $I / V=g$, the current distribution $P(I)$ can be converted to a conductance distribution via $P(g)=P(I / V)$ in a constant bias mode. Figures 3(a)3(d) show examples of conductance distributions. A few important features can be seen. First, for the same $x$ but different bias $V$ [Figs. 3(a), 3(b), $x=0.547, V=15.2 \mathrm{mV}$ and 26.7 $\mathrm{mV}$ ], the conductance distributions $P(g)$ are nearly identical, indicating that Ohm's law is applicable at a microscopic level. However, for different $x$, the distribution is different, as shown by Fig. 3(c), where $x=0.532$. For other samples and bias, the same results were obtained. Secondly, for all samples, the conductance distribution can be fitted by a power law as $g \rightarrow 0$, i.e.,

$$
P(g) \propto g^{-\alpha}, \quad 0<g<g_{b},
$$

where the exponent $\alpha$ depends on $x$, as illustrated by Fig. 4 . In order to show the distribution in the high-conductance region more clearly, a semilogarithmic scale was plotted (for $\left.g>g_{b}\right)$ in Fig. 3(d). The straight line in the plot demonstrates the third important feature, that is, $P(g)$ can be approximately described by an exponential distribution in the high conductance region.

The conductance distribution obtained by C-AFM is a measure of conductance at different locations at the top of the sample to the bottom. However, if the conductance is dictated by the microscopically high resistance region, $P(g)$ obtained via C-AFM should represent the microscopic $P(g)$ as assumed in the theories. ${ }^{8,9}$ Let us first examine the sources of experimental errors that influence the accuracy of the measurements. The main sources are contact resistance $R_{\mathrm{CR}}$ and the noise of the pre-amplifier. The relative noise in determining conductance $g$ is due to the current noise $\Delta g / g$ $\approx \Delta I / I$, where $\Delta I \approx \pm 1.5 \mathrm{pA}$. As an estimation, for the data presented in Fig. $3, \Delta g / g \leqslant 15 \%$ if $g>1 \times 10^{-9} \Omega^{-1}$. However, for $g<1 \times 10^{-9} \Omega^{-1}$, the low-conductance region in Fig. 3(c), $\Delta g / g$ is fairly large, which will give rise to a large uncertainty of the exponent $\alpha$.

The contact resistance $R_{\mathrm{CR}}$ results from the point contact of the tip and the CP's, which is in series with the CP's, and can be neglected according to existing theories. ${ }^{17}$ Detailed analysis shows that the measured $g \approx g_{t}(1+\bar{d} / L),{ }^{18}$ where $g_{t}$

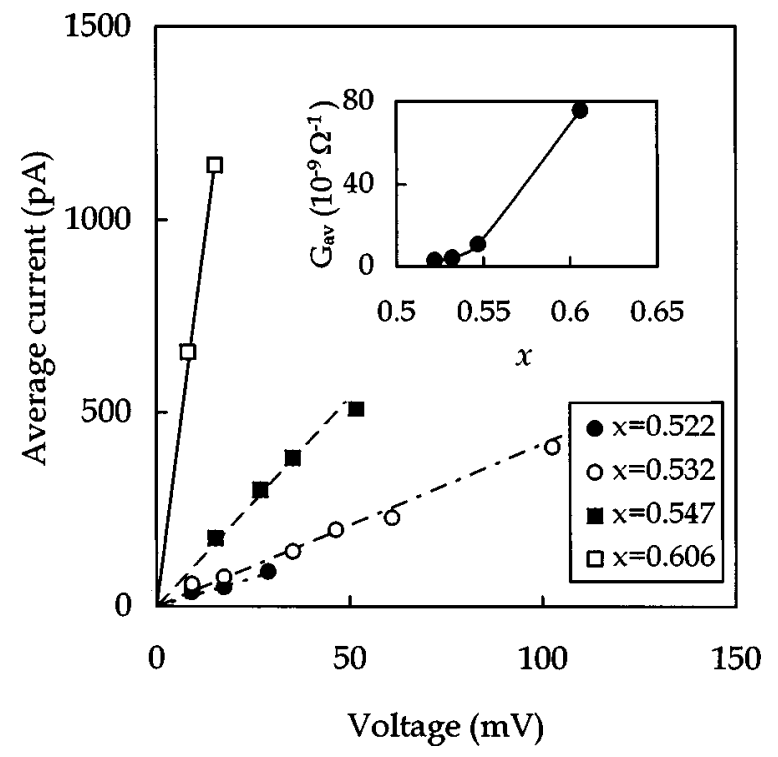

FIG. 2. Averaged $I-V$ characteristics for different samples and at different biases. Inset: the dependence of $G_{\mathrm{av}}$ on $x$. The solid line is a guide for the eye. 

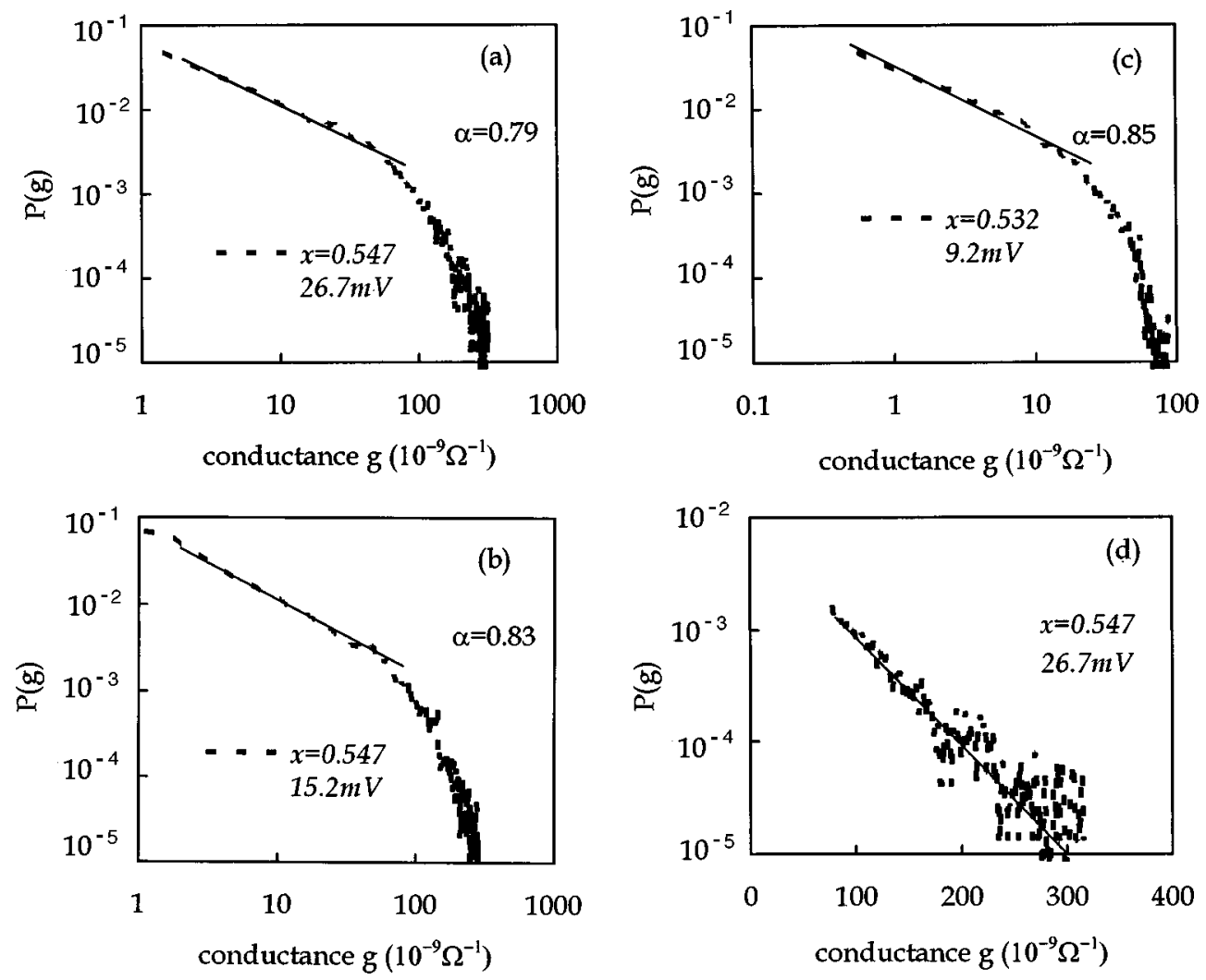

FIG. 3. Conductance distribution for samples with different $x$ or at different biases. (a) $x=0.547, V=26.7 \mathrm{mV}$. (b) $x=0.547, V$ $=15.2 \mathrm{mV}$. (c) $x=0.532, V=9.2 \mathrm{mV}$. The solid lines are fits of power law $P(g) \propto g^{-\alpha}$. For (d), the high-conductance region of Fig. 3(a) is re-expressed in a semilogarithmic plot and fitted with an exponential distribution by a solid line.

is the true conductance of the $\mathrm{CP}, \bar{d}$ is the size of the metal particles, and $L$ is the effective path length. Since $\bar{d} \ll L, g$ $\approx g_{t}$. Experimentally, $R_{\mathrm{CR}}$ depends on the loading force (pressure) in terms of the contact area and the possible pressure dependence of the conductance. Following Hertz's law, ${ }^{20,21}$ the contact radius $a=(3 F R / 4 E)^{1 / 3}$, where $F$ and $R$ denote the force and tip radius. $1 / E=\left(1-\nu_{1}^{2}\right) / E_{1}+(1$ $\left.-\nu_{2}^{2}\right) / E_{2}$, where $E_{i}$ and $\nu_{i}$ are the Young's moduli, Poisson ratios for tip and sample, respectively. By using the $E_{i}$ and $\nu_{i}$ of $\mathrm{Au}$ (tip) and $\mathrm{Ni}$ (sample), $R$ (taken as $50 \mathrm{~nm}$ ) and the $F$, the contact radius is $3.1 \mathrm{~nm}$ for the force of $70 \mathrm{nN}$, which is the same order as the metal particles size $\bar{d}$. The pressure is then $p=F / \pi a^{2}=2.3 \mathrm{GPa}$. Therefore, the sample is elastically contacted. ${ }^{20,21}$ Hence, the pressure- (plastic deformation) induced changes in conductance can be neglected. Systematic studies of the force dependence of conductance is in preparation. If the loading force is too low, the tip-surface tunneling effect will dominate the resistance. However, if the force is too large, it will degrade the lateral resolution. The true contact resistance was not calibrated. It was tested on the $\mathrm{Ni}_{x}\left(\mathrm{SiO}_{2}\right)_{1-x}$ sample with $x=0.78$, where most of the CP's are connected and tunneling plays a minor role. By using the same tip and same experimental conditions, the conductance of CP's cannot be measured in our experimental setup since even at a bias of $0.1 \mathrm{mV}$, the current of all CP's exceeded our current range of $10 \mathrm{nA}$. Because of this saturation, it was impossible to measure the conductance variations. If we assume that for $x=0.78$, the CP's behavior is similar to perfect metal and neglect their resistance, then $R_{\mathrm{CR}}$ (including the finite resistance of the tip itself) can be safely estimated at less than $10 \mathrm{~K} \Omega(0.1 \mathrm{mV} / 10 \mathrm{nA})$, which is several orders less than the measured resistance, as shown above. On the other hand, the local heating effect by the high current density (typically $10^{3} \mathrm{~A} / \mathrm{cm}^{2}$ at the contact point) may have influence on the measurement. However, the reproducibility indicated that there were no unrecoverable damages on the surface by the high current density. Macroscopic measurements revealed that the resistivity follows a weak- $\ln (T)(5-300 \mathrm{~K})$ dependence for all samples studied in

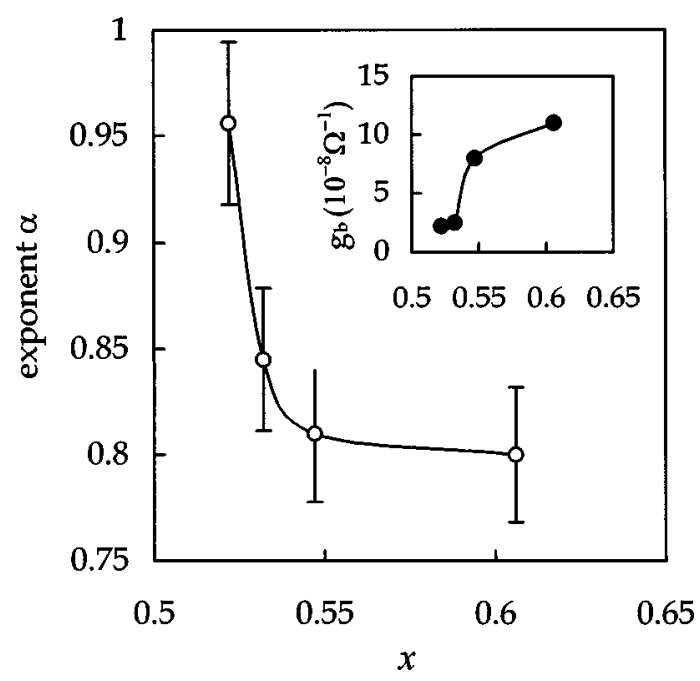

FIG. 4. The dependence of the exponent $\alpha$ on the volume fraction $x$ of the metallic phase. Inset: the boundary conductance $g_{b}$ vs $x$. The lines are guides for the eye. 
this study. 2,3,6 Therefore, the heating effects may have no significant effects on the conductance, although the details are not very clear.

To understand the power-law distribution in the lowconductance region, let us first review the existing continuous percolation models ${ }^{8,9}$ that could give rise to the powerlaw distribution. Both theories predict a distribution following $P(g) \propto g^{-\gamma}$ (as $\left.g \rightarrow 0\right)$, which leads to a nonuniversality of behavior in terms of the values of the critical exponents. In the well-known Swiss-cheese model, Feng et al. showed that the conductance distribution due to the narrow "necks" controls the connectivity, and that $\gamma=1 / 3$ for an equal distribution of "neck" size. "The measured value of $\gamma$ is close to 1 , indicating that the Swiss-cheese model fails to describe our system. In fact, from the thermodynamic point of view, the surface tension of nanometal particles is not in favor of forming narrow "necks"' in nanocomposites. It is interesting to note that a similar power-law dependence can also result from the tunneling as proposed by Balberg, ${ }^{9}$ with $\gamma=1-\delta / \Gamma$. Here, with tunneling, the exponent $\gamma$ is related to the ratio of the tunneling distance $\delta$ and the average distance of two adjacent conducting particles $\Gamma$. For a fixed $\delta$, the higher the $x$, the smaller the $\Gamma$ and $\gamma$, which is in agreement with the experimental results, as shown by Fig. 4. Hence, in our nanocomposite system, it is likely that the tunneling between isolated metal particles gives rise to a power-law distribution.

As shown by Fig. 3(d), for $g>g_{b}$, the conductance distribution can be approximately fitted by exponential distribution. One possible explanation is that it describes the conductance distribution of the connected conducting networks.
Firstly, our results qualitatively agree with the intuitive thought that the conduction via metallic percolation channels has to be localized in a relatively narrow region in the distribution. ${ }^{19}$ In other words, the difference in the conductance value should result from the difference in the length of the connected paths with essentially the same resistivity value, which is in agreement with the result as shown in Fig. 3(d). Secondly, as $x$ increases, there are more connected particles, and therefore $g_{b}$ will increase, as shown by the inset of Fig. 4. These qualitative features suggest that the exponential tail of the conductance distribution is due to metalliclike conduction via the connected particles. However, we could not exclude other possibilities, such as the possibility due to the tunneling between grain clusters. ${ }^{22}$

In conclusion, we have systematically studied the conductance distributions of the conducting paths in the percolating metal-insulator nanocomposites $\mathrm{Ni}_{x}\left(\mathrm{SiO}_{2}\right)_{1-x}$ thin films. We demonstrate the existence of a power-law distribution of the conductance probability as $g \rightarrow 0$, and of an exponential distribution in high-conductance regions. Moreover, the conductance satisfies the Ohm's law at the microscopic level. The results indicate that, in the vicinity of the percolation threshold, there is both tunneling conduction via isolated metal particles and metalliclike conduction via connected metal particles.

We have benefited from valuable discussions with Dr. P. Sheng, Dr. P. M. Hui, Dr. Z. Q. Zhang, Dr. M. B. Heaney, and Dr. A. B. Pakhomov. This work was in part supported by the Research Grant Council of Hong Kong via Grant Nos. CUHK501/92E, CUHK4137/97E, and HKUST692/96P.
*Electronic address: EZLUO@EE.CUHK.EDU.HK

${ }^{1}$ M. Fujimori, S. Mitani, and S. Ohnuma, Mater. Sci. Eng., B 31, 219 (1995).

${ }^{2}$ B. Zhao and X. Yan, Physica A 241, 367 (1997).

${ }^{3}$ A. B. Pakhomov, X. Yan, and B. Zhao, Appl. Phys. Lett. 67, 3497 (1995).

${ }^{4}$ Physical Phenomena in Granular Materials, edited by G. D. Cody, T. H. Geballe, and P. Sheng, MRS Symposia Proceedings No. 195 (Materials Research Society, Pittsburgh, 1990).

${ }^{5} \mathrm{P}$. Sheng (unpublished).

${ }^{6}$ X. N. Jing, N. Wang, A. Pakhomov, K. K. Fung, and X. Yan, Phys. Rev. B 53, 14032 (1996).

${ }^{7}$ X. N. Jing and X. Yan, Introduction to Wave Scattering, Localization and Mesoscopic Phenomena (Academic, Boston, 1995).

${ }^{8}$ S. Feng, B. Halperin, and P. N. Sen, Phys. Rev. B 35, 197 (1985).

${ }^{9}$ I. Balberg, Phys. Rev. Lett. 59, 1305 (1987).

${ }^{10}$ I. Balberg, Philos. Mag. B 56, 991 (1987).

${ }^{11}$ R. Viswanathan and M. B. Heaney, Phys. Rev. Lett. 75, 4433 (1995).

${ }^{12}$ E. Z. Luo, J. X. Ma, J. B. Xu, I. H. Wilson, A. B. Pakhomov, and
X. Yan, J. Phys. D 29, 3169 (1996).

${ }^{13}$ E. Z. Luo, J. B. Xu, W. Wu, I. H. Wilson, and X. Yan, Appl. Phys. A: Mater. Sci. Process. 66A, S1171 (1998).

${ }^{14}$ G. Xiao and C. L. Chien, Appl. Phys. Lett. 19, 1280 (1987).

${ }^{15}$ Y. Xu and X Yan, J. Mater. Res. 11, 2506 (1996).

${ }^{16}$ S. Ohnuma, H. Fujimori, S. Mitani, and T. Masumoto, J. Appl. Phys. 79, 5130 (1996).

${ }^{17}$ R. Wisendanger, Scanning Probe Microscopy and Spectroscopy (Cambridge University, Cambridge, England, 1994).

${ }^{18}$ We note that the radius of tip contact area and the mean free path of electrons are of the same order as the metal particle size $\bar{d}$ (nanometer range). Taking $g_{i}=\bar{d}^{2} /(\rho L)$, if either the Maxwell or Knudesen limit (Ref. 17) is applied, we will arrive at a similar result.

${ }^{19}$ Z. Q. Zhang (private communication).

${ }^{20}$ F. Houze, R. Meyer, O. Schneegans, and L. Boyer, Appl. Phys. Lett. 69, 1975 (1996).

${ }^{21}$ T. G. Ruskell, R. K. Workman, D. Chen, and D. Sarid, Appl. Phys. Lett. 68, 93 (1995).

${ }^{22}$ A. B. Pakhomov (private communication). 